\title{
Research on the Implementation of In-House Calibration in Automotive Testing Laboratory
}

\author{
Shimin Zhang ${ }^{1, a^{*}}$, Lixiong Zhang ${ }^{2, b}$ \\ ${ }^{1}$ China Automotive Technology and Research Center, china \\ ${ }^{2}$ China Automotive Technology and Research Center, china \\ azhagnshimin@catarc.ac.cn, bzhanglixiong@catarc.ac.cn
}

\begin{abstract}
Keywords: Testing Instrument; In-house Calibration; Measurement Traceability; Automotive Testing Laboratory

Abstract. In order to ensure the traceability of the instruments for automotive testing laboratory, the article describes the necessity and significance of in-house calibration in automotive testing laboratory, according to the CNAS- CL31 " Requirements for In-house Calibration " proposed five important aspects for automobile testing laboratory to carry out in-house calibration activities.
\end{abstract}

\section{Introduction}

The section 5.6.1 of CNAS-CL01 " Accreditation Criteria for the Competence of Testing and Calibration Laboratories" states that "all instruments used to detect and / or calibrate that have significant effect on the accuracy or validity of the results of testing, calibration and sampling results, comprises an auxiliary measuring instruments ( e.g. a device for measuring ambient conditions ), should be calibrated before being put into using." This shows that measurement traceability is the basic requirement for instruments and measurement. In automotive testing laboratory, some special instruments that used in narrow areas are difficult to find a metering mechanism that has the appropriate ability or accredited calibration agencies. So most of the testing laboratory use the in-house calibration method. February 2011, China National Accreditation Service for Conformity Assessment issued the CNAS-CL31: 2011 " Requirements for In-house Calibration", prescribed to the in-house calibration work in testing laboratory, in order to ensure the measurement traceability of the test results. Therefore, in order to meet the China National Accreditation Service for Conformity Assessment's requirement for instruments' traceability in testing laboratory, while ensuring the accuracy of data in testing and research activity, this paper discuss the key requirement for the implementation of in-house calibration in automotive testing laboratory.

\section{The Necessity of Conducting In-house calibration in Automotive Testing Laboratory}

2.1 There is no calibration standard in country

Automotive Testing Laboratory has some special cases. The application field of special testing instruments is relatively narrow, such as ozone aging test chamber, etc. These instruments have no calibration specification in country, and no recognition of calibration institutions to providecalibration services for these devices. The traceability of test results measurement cannot be guaranteed. And this kind of instruments measurement can be traceable to the international system of units through some way, so it is necessary for automotive testing laboratory to carry out in-house calibration activity for these instruments according to the instrument usage and specifications to select a suitable standard measuring instruments and formulate rational and effective calibration method for in-house calibration.

2.2 Large number of the same parameter testing instruments

Automotive testing laboratory has the same parameter and low-value consumable testing instruments, such as force sensor, acceleration sensor and so on. The quantity of this kind of instruments is larger. If the calibration of all these devices was carried out outside the automotive testing laboratory, according to the metrology department work schedules for calibration, it would be lead to the longer calibration cycle. Some calibration results will take a month or even longer time. It 
affects the normal conduct of the test, calibration fee is high accordingly, and increases the great waste of time and cost virtually. In order to shorten the calibration cycle, improve the use rate of detection instruments, get effective protection of automobile test. Such instruments is necessary to carry out in-house calibration.

2.3 Large number of big special instruments should not be moved and tested

Automotive testing laboratory has quite a large number of special instruments should not be moved and disassembled, such as the chassis dynamometer, large car test rig etc. Especially the calibration of large instruments, such instruments although has units of measurement with a calibration capability, but due to the plenty of measurement business, home time calibration services may be delayed. It needs to carry out in-house calibration to shorten the calibration cycle and improve the use rate of detection instruments.

\section{The Significance of In-house Calibration for Automotive Testing Laboratory}

3.1 Reduce operating costs, improve efficiency

The number of instruments in the automobile testing laboratory is various, especially some special or big instruments. Take the China Automotive Technology \&Research Center as an example, annual inspection and calibration costs amounted to nearly a million RMB. 2/5 of the amount of calibration is geometry, temperature, quality and other basic quantities. And these can be established by the establishment of laboratory standards, the establishment of internal calibration procedures, in order to achieve the traceability and meet the quality system requirements, thereby reducing the calibration cost of the automotive testing laboratory. On the other hand, the in-house calibration can greatly shorten the calibration cycle. The use of the instruments will not be delayed due to the long calibration period, and greatly improving the detection instruments utilization, improving the detection of production efficiency.

3.2 Improve the accuracy and validity of the detection traceability

The in-house calibration of automotive testing laboratory is beneficial to improve the accuracy and validity of the detection data. In automobile testing laboratory, the in-house calibration of the instruments can make the inspection engineer and the tester of the relevant test more in-depth understanding of the performance and principle of device, more skilled and steadily operating the instruments operation, in the same time make the testing data more accuracy. Effectively reduce the customer's query to the validity of testing data. The measurement uncertainty evaluation report that need to write after the in-house calibration can also provide the basis for the synthesis of the uncertainty of the inspection report. The test report can reflect the whole test result, and the relevant index of the sample can be correctly evaluated.

3.3 Be propitious to the integrity of the quality system for automotive testing laboratory

The measurement traceability of the instruments is one of the important elements of CNAS-CL01 " Accreditation Criteria for the Competence of Testing and Calibration Laboratories". A few years ago, the China National Accreditation Service for Conformity Assessment doesn't have rules for the validity and normalization of in-house calibration, and lack of guidance documents for the relevant accreditation review. The implemented of in-house calibration of some laboratory have irregularities, and even do not meet the requirements of the traceability of amount. To ensure the validity of the recognition, the in-house calibration activity is effectively regulated, the China National Accreditation Service for Conformity Assessment . finally released the CNAS- CL31 " Requirements for In-house Calibration ", in order to regulate the in-house calibration activity of the measuring instruments implemented in the laboratory, then make sure that the measurement results is valid. Some special inspection instruments in the laboratory can not be calibrated by the third party metrological mechanism, so the in-house calibration of the laboratory is needed. Only research on the in-house calibration and develop the conditions for implementation, can meet the requirements of the China National Accreditation Service for Conformity Assessment about in-house calibration . To ensure the conformity of the laboratory system, and the integrity of the quality system. 


\section{The Implementation of In-house Calibration in Automotive Testing Laboratory}

The personnel, measurement standards, calibration methods, calibration certificates, and the quality assurance of calibration results are the five key factors of the implementation of in-house calibration.

\subsection{Personnel}

It is required to train a number of in-house calibration personnel who can finish this work in automotive testing laboratory. The in-house calibration personnel should familiar with the testing instruments. According to the CNAS- CL31 " Requirements for In-house Calibration ",the in-house calibration personnel should have relevant training about knowledge of measure and calibration skills, and they should pass the exam and certified or authorized. Because the external training can't meet the calibration requirement of the special instruments for automobile testing, The training of personnel is required to take the form of external training and internal training and regular assessment. The training and assessment should include basic knowledge, such as calibration, period verification, uncertainty analysis and so on. For each in-house calibration member, the measurement parameters of the instrument and instruments should be trained to ensure that each of the in-house calibration personnel can master professional measurement knowledge and calibration requirements, in the same time to improve the professional quality of the in-house calibration personnel. Provided personnel guarantee for the development of in-house calibration for the automotive inspection laboratory.

4.2 Measurement standard

The traceability of measurement standards is the basic requirement for the effective traceability of in-house calibration. Selection of measurement standards for in-house calibration items for automotive testing laboratory, not only need to meet the requirements of CNAS-CL01" Accreditation Criteria for the Competence of Testing and Calibration Laboratories" and CNAS-CL06 "Requirements on the Traceability of Measurement Results" for measurement traceability, but also need to consider the nature of calibration project and laboratory specific circumstances, under the premise that the calibration method is required. The verification of uncertainty and the stability of the measurement standards, should ensure that the uncertainty of measurement standards is achieved by the calibration of the instrument to measure the uncertainty of the $1 / 2$ or even the same grade. A set of maintenance procedures is developed for the measurement standards to guarantee the credibility of the calibration status of the measurement standards.

4.3 Calibration method

The in-house calibration method is the key to the implementation of in-house calibration for automotive testing laboratory, and is the basic technical support for the measurement. Compilation of in-house calibration method to meet the requirements of measuring instruments, and meet the requirements of CNAS files, adhere to the practical principle and the principle of economic benefit, consider the demand of testing laboratories now and in the future. Article 4.5 of CNAS-CL31: 2011 " Requirements for In-house Calibration" clearly defined:" The standard method should be adopted preferentially for in-house calibration in the laboratory. When there is no standard method, the non standard method can be used, such as the method of self - use directed and the method of measurement instruments manufacturers recommended." The in-house calibration member shall determine whether directly refer to national standards or use self - preparation and calibration procedures. If there is no national metrological verification regulations or calibration specification for the calibration items, automotive testing laboratory should refer to the national or industry standards in the corresponding parameters and methods, or product brochures and other information to prepare for in-house calibration procedures. The in-house calibration procedure should be based on the requirements of the JJF-2000 "National Calibration Code for Measurement "and the nature of the internal calibration project. The in-house calibration procedure should be approved by the technical committee and approved by the technical director. Take China Automotive Technology and Research Center as an example, we have established five in-house calibration procedures, including displacement sensor, multi axis force sensor, acceleration sensor, chassis dynamometer and battery test system. 
4.4 Calibration certificate and uncertainty

The calibration record should be well calibrated after the completion of in-house calibration experiment, and the contents of the calibration record should be in accordance with the calibration method. Automotive testing laboratory can not issue a calibration certificate, but also issued a simplified calibration certificate. The laboratory shall carry out in-house calibration uncertainty evaluation. The calibration certificate should reflect the calibration results and the measurement uncertainty for calibration results. The laboratory shall analyze the calibration results, in order to determine whether the measuring range of the instruments can meet the detection activity, the correct of the instruments' indication value. At the same time, the effect of the uncertainty of the instruments is evaluated, which provides theoretical basis for determining the interval between the instruments maintenance, the time and the calibration cycle.

4.5 Proficiency testing

The CNAS-CL31 " Requirements for In-house Calibration " stipulates that" the laboratory shall seek and take appropriate proficiency testing activities, in order to control the quality of the implementation of in-house calibration. If possible, these proficiency testing activities should meet the requirements of CNAS- RL02 "Rules for Proficiency Testing". The calibration procedure of the automotive testing laboratory is required to carry out proficiency testing activities. Proficiency testing work give priority to use the method of comparative transmission between laboratory. The measured object is measured with the measured standard of the examination. And then measure the object with another higher measurement standard. Secondly, the comparison between laboratory can be taken. It is assumed that the measurement criteria of each laboratory have the same accuracy level, and the average of the measured results obtained by each laboratory is used as the best estimated value of the measurement. If the above two methods are unable to achieve,the method of comparing with two devices or two methods in the same laboratory can be used.

\section{Conclusion}

In conclusion, The in-house calibration of automobile testing laboratory is the inevitable trend of adapting to the development and management of the modernization of laboratory. The automobile testing laboratory should continuously improve the ability of management calibration technology, discover the problem and solve the problem in time.Under the premise of conforming to CNAS-CL31: 2011 " Requirements for In-house Calibration",improve the management system of in-house calibration and provide reliable measurement guarantee for automobile testing laboratory to carry out testing work effectively and accurately, and ensure the traceability of a quantity value of the testing instruments especially special instruments in narrow applicated field for automobile testing laboratory.

\section{References}

[1] CNAS-CL01:Accreditation Criteria for the Competence of Testing and Calibration Laboratories.2005

[2] CNAS-CL31: Requirements for In-house Calibration.2011

[3] JJF 1071-2000, The Rules for Drafting National Calibration Specification.2000

[4] Li Zaichun.Study on the Internal Calibration in Testing Laborary. Measurement and Testing Technology , 2013 ( 40 ) $2: 81-82$ 